\title{
Le droit maritime international s'applique-t-il aussi au domaine de la santé en Suisse?
}

J'ai obtenu mon brevet de voile pour la haute mer il y a vingt-cinq ans - et appris le droit maritime en même temps que la navigation. Un principe m'a particulièrement impressionné:

Lorsqu'un bateau est en détresse et qu'il doit être remorqué ou mis en sécurité, il est absolument déterminant de savoir qui remet la corde ou le câble de remorquage. Si la corde vient du «navire en détresse», l'indemnité de sauvetage est une question de négociation, donc d'un accord entre capitaines. Si la corde vient du remorqueur (donc du bâtiment qui vient en aide), le bateau sauvé peut être pris à titre d'indemnité de sauvetage. L'embarcation et la marchandise qu'elle transporte sont alors remises en gage au sauveteur. Le petit détail de savoir qui jette la corde ou la reçoit décide, dans cette situation, entre les biens de l'un et de l'autre.

Deux événements récents m’ont fait repenser à cette coutume maritime: l'un d'eux est la campagne d'information «Carepay» menée par quelques assureurs. Nos patients sont appelés par lettre personnelle et en des termes émouvants à intervenir auprès de nous, médecins, pour demander que la facture soit envoyée directement à l'assureur.

L'autre événement a eu lieu sous la forme d'un article récemment paru dans la revue «Dosis», organe de la Société suisse de pharmacie. Le pharmacien y est défini comme étant le premier point de contact idéal (ou «gatekeeper») en santé publique. Selon l'article, le monopole du diagnostic, réservé aux médecins, devrait être assoupli par «l'attribution au pharmacien d'une compétence en matière d'ordonnances pour les cas bénins».

Chère lectrice, cher lecteur, vous avez raison: nous sommes une nation continentale, nous ne connaissons pas de naufrages maritimes et n'avons pas besoin de nous préoccuper de cordages. Pourquoi donc évoquer cette image?

La proposition dite «Carepay» porte sur l'échange des données électroniques de facturation entre corps médical et assureurs, une situation qui en ce moment est bloquée. Il serait facile de la débloquer, de travailler en synergie, de trouver une solution satisfaisante pour tous. Et il est inutile de réagir obstinément en désécurisant les patients et en voulant abolir le tiers garant. Toutefois, la question de savoir qui fait quelle offre, et qui donne la corde, n'est apparemment pas indifférente.

Le problème de la relève chez les médecins de premier recours est évident. Et les pharmaciens ont leur place historique et incontestée dans les soins fournis à la population. Il convient également de relever la saine rivalité qui existe entre ces deux professions et leurs représentants. Là aussi, il vaut la peine de réfléchir aux possibilités d'œuvrer en synergie, notamment dans le cadre des systèmes de gestion des soins. Toutefois, la revendication des pharmaciens de pouvoir rédiger eux-mêmes des ordonnances est «une offre d'assistance» qu'il faut rejeter poliment mais très clairement.

Depuis ma première leçon de navigation et de droit des mers, j'ai de nombreuses lieues à mon actif et le plaisir de la mer ne me quitte pas. En tant que barreur de voilier, j'ai beaucoup appris - et je me sers aussi de ces connaissances dans mon travail et dans la politique de santé.

Dr Olivier Kappeler, membre du Comité central de la FMH 